\title{
Modeling of Potassium Chloride Sensor with Two Dimensional Optical Nanostructure
}

\author{
Santosh Kumar Sahoo \\ Assoc. Professor, CVR College of Engineering / EIE Department, Hyderabad, India \\ santosh.kr.sahoo@gmail.com
}

\begin{abstract}
Presence of potassium chloride concentration in aqueous solution is investigated using optical sensor. Optical sensor is modeled by two dimensional optical nanostructure or photonic crystal structure, which made from silicon materials (background/ substrate) consists of $8 \times 8$ air holes containing aqueous potassium chloride solution. Here measurement techniques centered on logarithmic difference of photonic band gap in regard to unlike concentrations of potassium chloride. The photonic band gap is found by means of plane wave expansion scheme. Proposed model is scheduled to quantify the output voltages with respect to same concentrations. Simulation result revealed that potential is measured at photodetector varies logarithmically with respect to potassium chloride concentration, which leads to an accurate measurement of potassium chloride concentration in aqueous potassium chloride solution.
\end{abstract}

Index Terms: Optical nanostructure, Potassiumchloride, Substrate, Concentration, Photo-detector

\section{INTRODUCTION}

Optical nanostructure or photonic crystals (PC) are composed of periodic dielectric nanostructures which affect the motion of photons in the same way as the periodicity of semiconductor crystal affects the motion of electrons [1]. As this structure is a repeatedly sections of high and low dielectric constant so the possibility of photons propagating through this structure depends on wavelength. Wavelength of a stream of photons which are allowed to travel is termed as mode and similarly rejected bands of wavelength photons are termed as band gap (BGP) [2]. Depending on dielectric structure's dimensions, varieties of photonic crystals are constructed such as 1D, 2D and 3D [3]. We restricted this paper to 2D Optical nanostructure. Insofar as review on optical sensor using PC is concerned, Author in reference [4] covenants with two dimensional PC structure for investigation of Sugar, Salt and Alcohol, again in reference [5] percentage of PAM hydrogel in an aqueous solution is investigated. In the same way in reference [6], author measures glycerol concentration in blood hemoglobinglycerol solution using three dimensional PC structure. Here glycerol measurement is performed by considering $3 \times 3$ air holes on substrate materials. But in the proposed work the concentration of potassium chloride concentration is investigated using two dimensional PC structure consisting of $8 \times 8$ air holes on silicon substrate. To obtain such concentration, such as a parameter, photonic band gap of above-mentioned crystal structure plays an important role. The $\mathrm{BG}_{\mathrm{P}}$ of two dimensional structure (PCS) can be calculated by plane wave expansion (PWE) [7], Finite difference time domain (FDTD) [8], Order 'N' spectral [9],
KRR [10] etc. Among these schemes PWE gives more precise result, because it offers an adequate information for matching boundary conditions in interface problems [11]. So we use the PWE method to calculate the band structure using Eigen values of the Maxwell's equation, henceforth solve Eigen frequencies for each of the propagation direction of the wave vectors. Potassium chloride is a natural compound in mineral, food and sea water which is highly necessary for various body functions like treatment of arterial hypertension [13]. Potassium is very important because it stimulates the kidneys to remove poisonous body wastes [14], for treatment of hypokalemia [15], treatment of animal diseases [16] and manufacturing of fertilizers [17]. The projected work is organized through PWE, discussed in section-2, simulation results along with discussions in section- 3 and conclusions narrated in section- 4 .

\section{Methodology}

The proposed structure for investigation of potassium chloride concentration is a square type two dimensional PC structure as shown in figure-1.

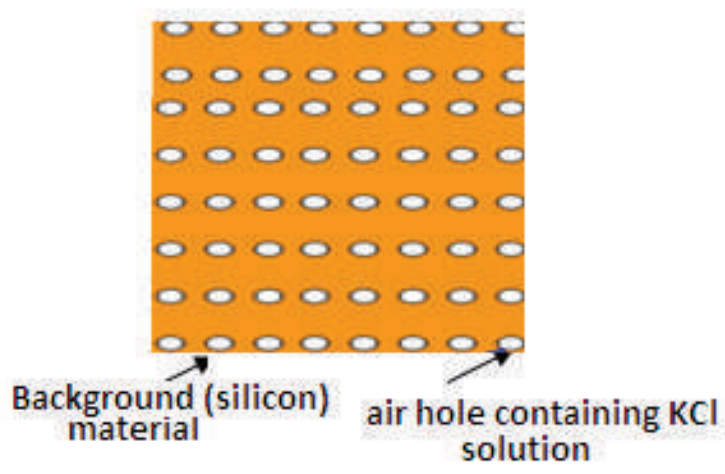

Figure 1. 2D photonic crystal hole with radius 0.42 micrometer and lattice constant 1 micro meter.

Figure-1 denotes a square lattice 2D photonic crystal structure with silicon as substrate, on which $8 \times 8$ air holes are etched. The radius of air holes is $0.4 \mu \mathrm{m}$ with lattice constant of $1 \mu \mathrm{m}$. The $\mathrm{BG}_{\mathrm{P}}$ of material changes by dielectric changes in air holes. Besides this, the same can be differs with variation of hole radius, lattice spacing or both. During the course of investigation, the light propagation in $2 \mathrm{D}$ photonic structure along $\mathrm{Z}$ axis and radiation has $\mathrm{TE}$, then 
the Helmoltz equation for the said field can be represented by considering Maxwell's equation as

$$
-\left(\frac{\partial}{\partial x} \frac{1}{\left(r_{11}\right)} \frac{\partial}{\partial x}+\frac{\partial}{\partial y} \frac{1}{\left(r_{11}\right)} \frac{\partial}{\partial y}\right) H_{z}(r)=\frac{\varpi^{2}}{c^{2}} H_{z}\left(r_{11}\right) \ldots
$$

Here $r_{11}$ is a $2 \mathrm{D}$ vector in $\mathrm{XY}$ plane.

For simulation the $\mathrm{BG}_{\mathrm{p}}$ is computed by using the equation-2 which is developed by simplifying the equation-1

$X\left(G_{11}\right)=2 f\left(\frac{1}{\epsilon_{1}}-\frac{1}{\epsilon_{2}}\right) \frac{J_{1}\left(G_{r_{11}}\right)}{G_{r_{11}}}$.

$J_{1}\left(G_{r_{11}}\right)$ is the Bessel's first order function. Here the limit is varies from $-\pi / T \ldots \ldots \ldots \pi / T, \quad G$ and $\quad G^{\prime}$ within $-2 \pi N / T \ldots \ldots .2 \pi N / T$, Where $(2 \mathrm{~N}+1)$ is the number of plane waves taken into account.

\section{Simulation ReSUlts ANALySiS}

Here band-gap of 2D photonic crystal structure plays an important role to find out the densities of Potassium chloride in potassium chloride solution. The photonic band-gap of the proposed structure depends on structure types, material background, air hole materials, air hole radius and air hole numbers etc. Table-1 and Table- 2 represent the complete figures regarding dielectric constant changes with different potassium chloride concentration [18].

TABLE I.

VARIOUS INPUTS FOR B $\mathrm{G}_{\mathrm{P}}$ SIMULATION

\begin{tabular}{|c|c|}
\hline STRUCTURE & SQUARE LATTICE \\
\hline Substrate & Silicon \\
\hline Air hole radius & 0.40 micro meter \\
\hline Lattice spacing & 1.01 micro meter \\
\hline
\end{tabular}

TABLE II.

Dielectric Constant Versus \% OF Potassium ChLoride

\begin{tabular}{|c|c|}
\hline$\%(\mathrm{gm} / \mathrm{ml}) \mathrm{KCl}$ in solution & Dielectric constant \\
\hline 2.51 & 1.8041 \\
\hline 5.01 & 1.8242 \\
\hline 7.01 & 1.8332 \\
\hline 10.02 & 1.8461 \\
\hline 16 & 1.8590 \\
\hline 20 & 1.8730 \\
\hline
\end{tabular}

Considering the information from Table-1 and Table-2, the PWE scheme is followed for computation of $\mathrm{BG}_{\mathrm{P}}$ of two dimensional structure having various potassium chloride concentration. The simulation outcomes for the same sample concentration of $2.5 \mathrm{gm} / \mathrm{ml}$ (dielectric constant is 1.804) and $20 \mathrm{gm} / \mathrm{ml}$ (dielectric constant is 1.873) are shown in figure 2(a) and 2(b).
Figure 2(a) represents the analytical outcome for $\mathrm{BG}_{\mathrm{P}}$ of two dimensional PC structure containing $2.5 \%$ of Potassium chloride in an aqueous solution.

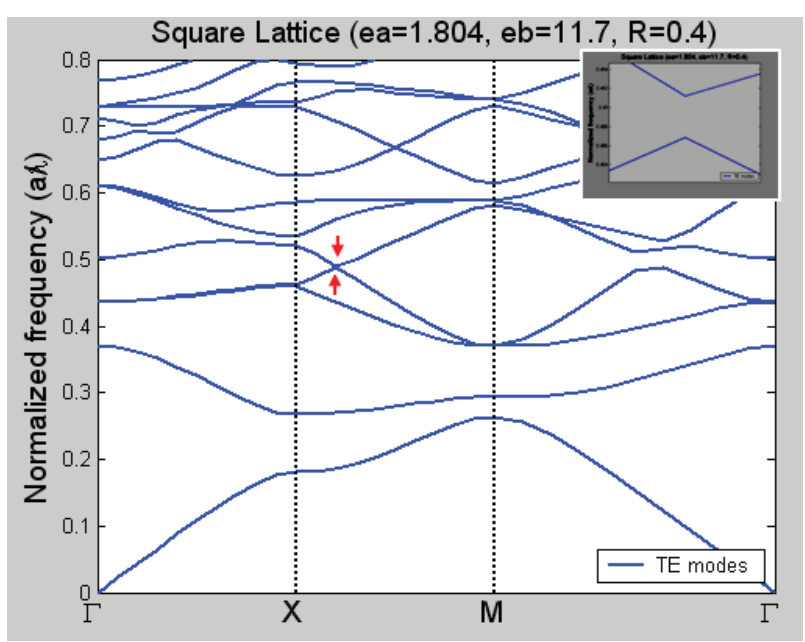

Figure 2. (a). BG simulation for $2.5 \%$ potassium chloride concentration

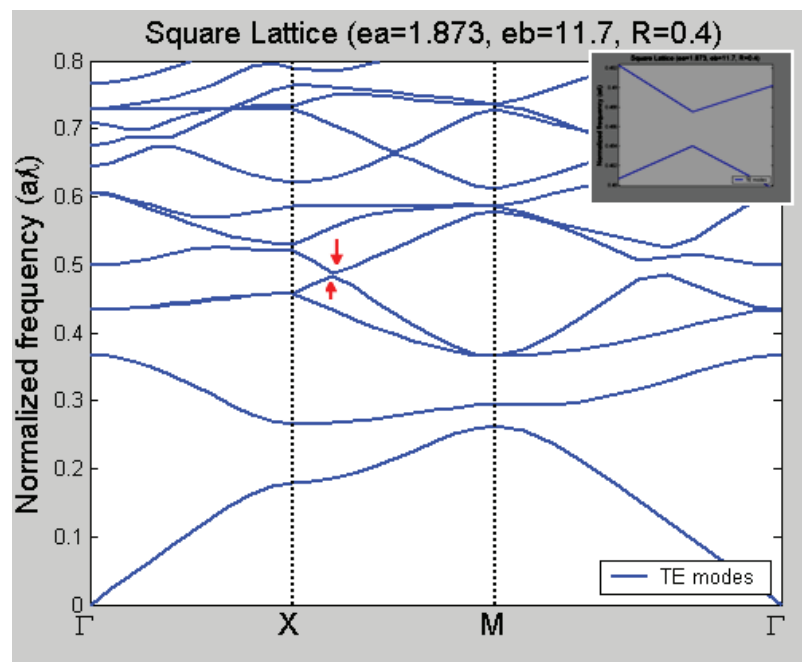

Figure. 2 (b). $\mathrm{BG}_{\mathrm{P}}$ simulation for $20 \%$ potassium chloride concentration

Figure 2(b) forecasts the effect of $\mathrm{BG}_{\mathrm{P}}$ of two dimensional PC structure containing $20 \%$ of Potassium chloride in an aqueous solution.

From figure 2(a) and 2(b), normalized frequency is shown between arrow marks and also zoomed. The zoomed figure is insert in each figure. Corresponding each normalized frequency, photonic bandgap is determined. The simulations are also done for other concentrations of potassium chloride such as $5 \mathrm{gm} / \mathrm{ml}$ (dielectric constant is 1.824), $7 \mathrm{gm} / \mathrm{ml}$ (dielectric constant is 1.833), $10 \mathrm{gm} / \mathrm{ml}$ (dielectric constant is 1.846 ), $16 \mathrm{gm} / \mathrm{ml}$ (dielectric constant is 1.859), but these results are not shown here.

On analyzing above photonic band-gap results, it is observed that $\mathrm{BG}_{\mathrm{P}}$ declines from $5.5 \mathrm{meV}$ to $4.4 \mathrm{meV}$ as concentration of potassium chloride increases from 2.5 $\mathrm{gm} / \mathrm{ml}$ to $20 \mathrm{gm} / \mathrm{ml}$. 
Graph is plotted between the photonic band gap and a different percentage Potassium chloride solution exposed in figure-3.

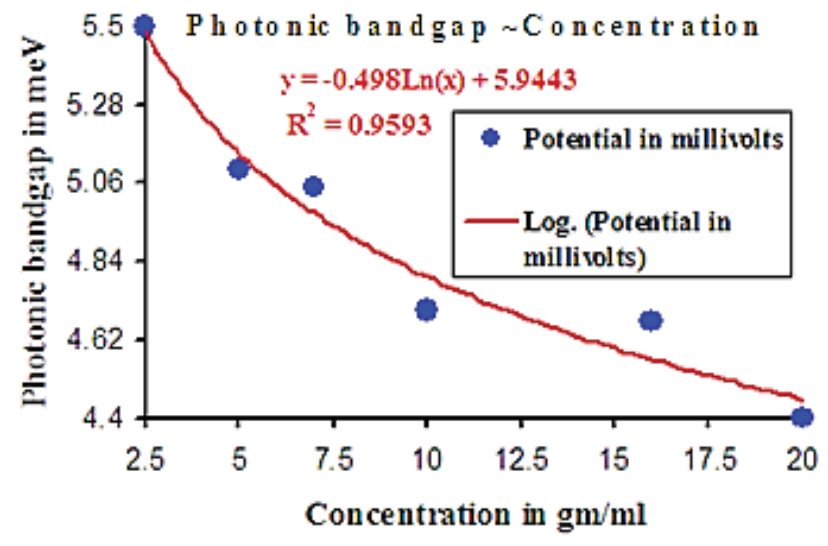

Figure 3. Photonic Band gap versus percentage of potassium chloride solution

From figure 3, it is seen that there is a logarithm variation between photonic band gap and concentration. Apart from this, it is observed that the decreasing variation is well builtin with logarithm trend line. This logarithm variation $\left(\mathrm{R}^{2}=0.9593\right)$ of photonic band gap and concentration gives sensing application for determining the potassium chloride concentration by 2D PC structures. For experimental measurement, Sodium D-lines concentration with wavelength $589 \mathrm{~nm}$ is incident at one end of the crystal and the potential is detected at other end. Experimental set up for concentration measurement is shown in figure-4.

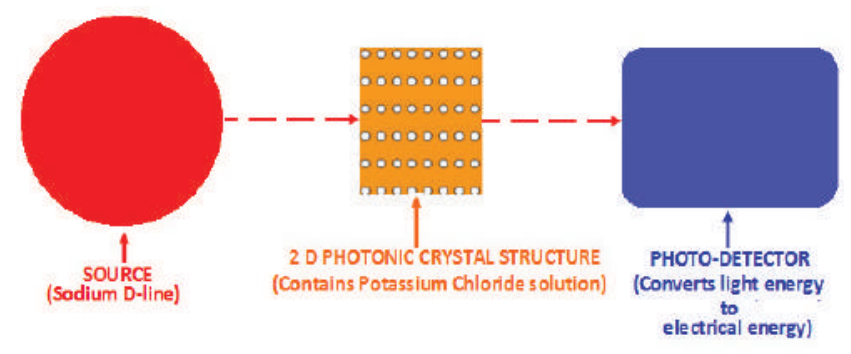

Figure 4. Experimental set up for concentration measurement

In this figure, it is seen that potentials at photo detector are determined with respect to concentration of potassium chloride. Using these results (voltage), a graph is plotted between potassium chloride concentrations along $\mathrm{X}$-axis and voltage in $\mathrm{Y}$-axis which is shown in figure-5.

Form Figure-5, it is found that potential decreases from $2.045 \mathrm{~V}$ to $2.0956 \mathrm{~V}$ as potassium chloride concentration increases in the range of $2.5 \mathrm{gm} / \mathrm{ml}$ to $20 \mathrm{gm} / \mathrm{ml}$. Reason for increase of potential with respect to potassium chloride concentartion is that reflectance (photonic bandgap) decreases from $5.5 \mathrm{meV}$ to $4.4 \mathrm{meV}$. Apart from this, it is observed that the change of output voltage is an remarkably trim with logarithim trendline $\left(\mathrm{R}^{2}=0.985\right)$. This outstanding logarithim deviation hints a precise measurement of potossium chloride concentration in aqueous potassium chloride solution.

\section{Potential $\sim$ Concentration}

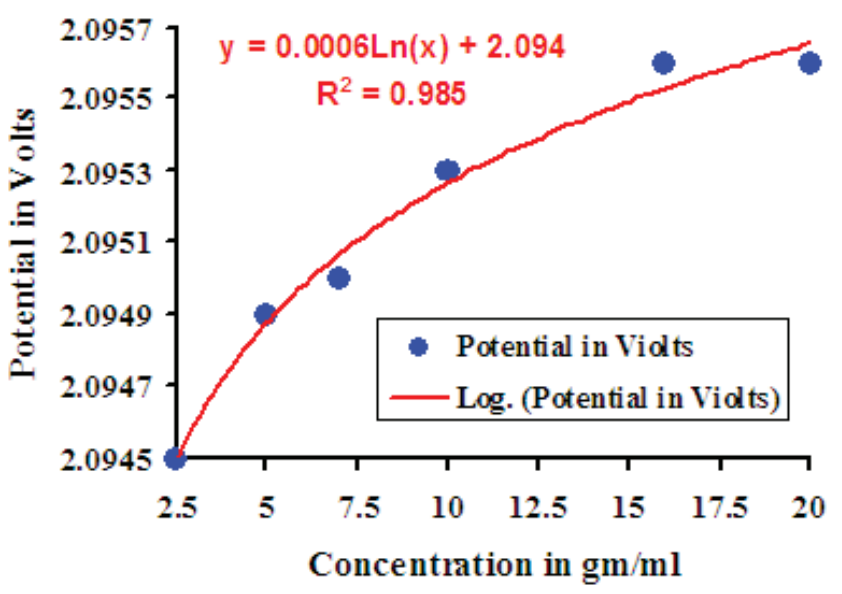

Figure 5. Potential versus concentration of potassium chloride

\section{CONCLUSIONS}

A Concentration of potassium chloride in their aqueous solution is investigated by using two dimensional photonic crystal structures. The experimental set up is also presented to measure the same concentration. It is found that both photonic band gap of photonic crystal structure and potential at photo detector varies logarithmically with respect to concentration of potassium chloride. Since measurement is carried out at optical frequency, this method can be used for accurate sensing application.

\section{REFERENCES}

1. E.Yablonovitch," photonic bandgap structures" : J.Optical Soc. America B 10(2),283-295(1993)

2. V. P. Bykov, "Spontaneous emission from a medium with a band spectrum Quantum Electronics 1975, 4:7, $861-871$

3. J.Joannopoulos, Steven G. Johnson, J.Winn, R D. Meade Photonic crystals: Molding the flow of light (Princeton University Press 1995)

4. G.Palai, S.K.Tripathy; A novel method for measurement of concentration using two dimensional photonic crystal structures; Optics Communications, Volume 285, Issues 10-11, 15, Pages 2765-2768, May 2012.

5. G.Palai, S.K.Tripathy, T.Sahu A novel technique to measure the sucrose concentration in hydrogel sucrose solution using two dimensional photonic crystal structures; Optik - International Journal for Light and Electron Optics; Volume 125, Issue 1,pages: 349-352, January -2014.

6. Measurement of glycerol concentration in B-H-G solution using 3D photonic crystal structure, Optik International Journal for Light and Electron Optics, Volume 125, Issue 12, June 2014, Pages 2875-2879

7. Xue-liang Kang, Guo-jun $\mathrm{Li}$, and Yong-ping $\mathrm{Li}$, Positive-negative refraction effect based on overlapping bands in a two-dimensional photonic crystal, JOSA B, Vol. 26, Issue 1, pp. 60-63 (2009) 
8. Kane Yee, Numerical solution of initial boundary value problems involving Maxwell's equations in isotropic media, IEEE Transactions on Antennas and Propagation 14, 302(1966)

9. P. Ordejon: Order-n tight-binding methods for electronicstructure and molecular dynamics Computational materials science 12, 157(1998).

10. Dunne, J.Lavon : Nutrition Almanac, (McGraw-Hill New York 1990)

11. Young-Chung Hsue, Arthur J. Freeman, and Ben-Yuan $\mathrm{Gu}$; Extended plane-wave expansion method in threedimensional anisotropic photonic crystals; Physical Review B; condensed matter and materials physics; Phys. Rev. B 72, 195118 - Published 30 November 2005

12. I.A. Sukhoivanov, I.V. Guryev, Physics and Practical Modeling: Photonic Crystals, Springer, Heidelberg, 2009.

13. W. L. T. Addison, The Use of Sodium Chloride, Potassium Chloride, Sodium Bromide, and Potassium Bromide in Cases of Arterial Hypertension which are Amenable to Potassium Chloride, Can Med Assoc J. Mar 1928; 18(3): 281-285.

14. Material Safety Data Sheet - Potassium Chloride. Sigma-Aldrich. July 2001.

15. Hypokalemia: Treatment \& Medication. Emedicine.medscape.com. Retrieved on 2012-02-16

16. http://www.petmd.com/pet-medication/potassiumsupplements

17. Lorient, Denis; Linden, G. (1999). New ingredients in food processing: biochemistry and agriculture. Boca Raton: CRC Press. p. 357

18. D.P. Subedi, D.R.Adhikari, U.M.Joshi, H.N.Poudel: Kathmandu Journal of Science, Engineering and Technology 2, 1 (2006) 